We Previously piloted ESC in several disease groups. The work Demonstrated that timely supportive care improves patient experience and reduces hospital admissions.

Incorporation of ESC within experimental cancer medicine is new in the UK. The aim is to help maximise patient recruitment and retention and enhance the patient experience within the context of experimental cancer medicine clinical trials (Phase I and non-randomised Phase II clinical trials).

Methods A Joint clinic was set up between the Experimental Cancer Medicine Team (ECMT) and the Supportive Care Team (SCT). These clinics are staffed by consultants from each team, research nurses and fellows and a clinical nurse specialist in supportive care. Patients being considered for or currently participating in a clinical trial are offered early referral to the supportive care team for assessment and management.

Results The pilot project began in September 2015. To date the SCT have undertaken 132 patient consultations within the ECMT. The predominant referral has been for optimisation of pain control, which is managed with specific consideration of the restrictions in the concomitant medication prescribing within Phase I trials.

Conclusion The ECMT at The Christie is the first early phase clinical trials unit to adopt ESC into practice. The ESC approach is now a routine part of the ECMT assessments of trial patients. Next steps will be to measure the impact of ESC on patient experience, eligibility for clinical trials, and admission avoidance.

\section{P-95 THINKING AHEAD: DISCUSSING RESUSCITATION DECISIONS WITH DIVERSE COMMUNITIES}

${ }^{1}$ Lucy Taylor, ${ }^{2}$ Zoebia Islam, ${ }^{1}$ Helen Eborall, ${ }^{2}$ David Miodrag, ${ }^{2}$ Wendy Gamble, ${ }^{2}$ Christina Faull. ${ }^{1}$ University of Leicester, Leicester, UK; ${ }^{2}$ LOROS, Hospice Care for Leicester, Leicestershire and Rutland, UK

\subsection{6/bmjspcare-2017-00133.94}

Background Improving Advance Care Planning (ACP) and increasing its equitable access is a key UK government strategy. Evidence (mainly from the USA) suggests that there are lower levels of ACP in people from Black, Asian and Minority Ethnic (BAME) communities. People from some BAME groups also seem to be more likely to desire invasive medical interventions, regardless of prognosis and impact on quality of life. The beliefs, values and needs of BAME communities in the UK have not been explored to see if the UK model of decision making around resuscitation 'fits' for them. Also, healthcare professionals (HCPs) report that they do not feel confident in providing culturally appropriate care for BAME patients and families in this situation.

Aims To identify barriers and enablers to HCPs discussing deterioration and resuscitation with patients and families from BAME communities, and to identify associated HCP training needs.

Methods Qualitative semi structured interviews with $30 \mathrm{HCPs}$. Results HCPs' interviews identify key barriers and facilitators of resuscitation discussions including differing attitudes towards death; differing values amongst different generations of migrants; and the need to find a balance between the desire to act in a non-discriminatory way whilst respecting cultural differences. Analysis of interviews informed a set of statements regarding decision making about resuscitation, which are being used as part of a ranking exercise (using Q methodology) with members of the public.

Conclusion There are significant barriers for HCPs when discussing resuscitation decisions with people from BAME communities. This increases the complexity of navigating ACP and achieving patients' preferences.

\section{P-96 THE MANAGEMENT OF TA - SURVEY OF NURSES WORKING IN A SPECIALIST PALLIATIVE CARE UNIT}

Jillian Wall, Sarah Awan. Royal Derby Hospital, Derby, UK

\subsection{6/bmjspcare-2017-00133.95}

Background National formularies recommend a step wise approach to Terminal Agitation (TA) using benzodiazepines and anti-psychotics.

Within our 21 bed specialist palliative care unit, a flowchart for management of TA was created in response to reviews where medical and nursing staff felt TA had been poorly controlled.

Four months after the introduction of the local guidelines, we conducted a spot survey of trained nursing staff to ascertain confidence toward management of TA.

Methods A standardised pro forma of 13 questions was used. Staff ranked confidence with various aspects of TA management using a numerical rating scale $(1=$ lowest, to $10=$ highest). Free text responses were collected regarding the most rewarding and challenging aspects of managing TA.

Results 11 of 12 nurses (all female) responded. Mean age 42 years (25-56), with a mean of 15 years since qualification

\begin{tabular}{|c|c|c|c|c|c|c|c|}
\hline $\begin{array}{l}\text { Years working in } \\
\text { palliative medicine }\end{array}$ & $\begin{array}{l}\text { Number of } \\
\text { timesmanaged TA }\end{array}$ & $\begin{array}{l}\text { Confidence in } \\
\text { recognising TA }\end{array}$ & $\begin{array}{l}\text { Confidence in } \\
\text { using midazolam }\end{array}$ & $\begin{array}{l}\text { Confidence in using } \\
\text { haloperidol }\end{array}$ & $\begin{array}{l}\text { Confidence in using } \\
\text { levomepromazine }\end{array}$ & $\begin{array}{l}\text { Confidence in using } \\
\text { phenobarbital }\end{array}$ & $\begin{array}{l}\text { Overall confidence in } \\
\text { managing TA }\end{array}$ \\
\hline 6.5 & $>20$ & 8 & 10 & 10 & 10 & 1 & 10 \\
\hline 5 & $>20$ & 8 & 9 & 5 & 8 & 0 & 8 \\
\hline 31 & $>20$ & 9 & 9 & 0 & 9 & 0 & 9 \\
\hline 3 & $>20$ & 8 & 10 & 8 & 10 & 7 & 6 \\
\hline 2 & $>20$ & 10 & 9 & 9 & 9 & 0 & 9 \\
\hline bank & $0-5$ & 2 & 2 & 2 & 1 & 1 & 2 \\
\hline$<1$ & $10-20$ & 6 & 8 & 5 & 7 & 1 & 6 \\
\hline 2.5 & $>20$ & 10 & 10 & 10 & 10 & 1 & 10 \\
\hline 18 & $>20$ & 9 & 9 & 9 & 9 & 1 & 9 \\
\hline 30 & $>20$ & 10 & 10 & 10 & 10 & 1 & 10 \\
\hline 8 & $>20$ & 6 & 7 & 7 & 7 & 0 & 7 \\
\hline
\end{tabular}


$(<1-36)$, and 10 years working in palliative medicine ( $<1$ year -31 years, one working as bank).

Most reported confidence in recognising and managing TA as well as the specific use of midazolam, haloperidol and levomepromazine. Respondents were less familiar with phenobarbital (table).

Lowest levels of confidence in managing TA were reported in respondents with the least experience of this.

Free text replies indicated that making patients settled was the most rewarding aspect of managing TA; impact on family was another common theme.

Respondents however described a number of challenges including difficulty in recognising $\mathrm{TA}$, and regarding medication decisions.

Conclusions With support of a local guideline, high levels of confidence were reported in use of first and second line medications for TA. However respondents still reported challenges in its management.

Future considerations We recommend regular education and training alongside ongoing reflection amongst medical and nursing teams after events of TA, to ensure best practice, team well-being and confidence with guidelines.

\section{P-97 EXPLORATION INTO USE OF CONTINUOUS SUBCUTANEOUS LEVETIRACETAM WITHIN PALLIATIVE CARE}

${ }^{1}$ Matthew Dore, ${ }^{1}$ Clare Marlow, ${ }^{2}$ WM CARES, Sharon Twigg. ${ }^{1}$ The Royal Wolverhampton NHS trust, West Midlands, UK; ${ }^{2}$ West Midlands Collaborative Actioning Research in End-oflife and Supportive Care, West Midlands, UK

10.1136/bmjspcare-2017-00133.96

With advances in medicine and people living longer with chronic medical conditions new subsets of patients emerge. One such subset is a group of patients for which seizure control is paramount yet they are not actively dying and their oral and intravenous route of administration has become unavailable/inappropriate. Traditional treatment for seizure management has been subcutaneous (S/C) midazolam, however this often does not balance Quality of Life (QOL) favourably for this interim period, primarily due to associated drowsiness. There have been numerous case reports using continuous subcutaneous infusion (CSCI) levetiracetam as an alternative for this group of patients, as a consequence of the perceived more favourable side effect profile.

We have collated the research published to date which outlines the appropriate scenarios and limitations of levetiracetam $\mathrm{S} / \mathrm{C}$ route (either intermittent or $\mathrm{CSCI}$ ). We have outlined the side effects, dose regimens and practical issued regularly encountered.

We have sought expert neurological advice and explored the limitations of other anti-epileptics via the $\mathrm{S} / \mathrm{C}$ route and noted hopeful upcoming newer therapies such as Brivaracetam and Lacosamide.

We have made recommendations regarding starting doses, disease aetiology considerations and practical titration and conversion issues.

Within this area of rapidly progressing research, collating what has been studied so far and gaining support out-with specialist palliative care organises our thoughts and creates an overview on which we can base future research and develop guidelines to encourage consistent safe practice.

\section{P-98 PATIENT AND CAREGIVER EXPERIENCES OF DO NOT ATTEMPT CARDIOPULMONARY RESUSCITATION (DNACPR) CONVERSATIONS: AN INTEGRATIVE REVIEW OF THE LITERATURE}

${ }^{1}$ Emma Carduff, ' ${ }^{1}$ Jean Lugton, ${ }^{1}$ Juliet Spiller, ${ }^{3}$ Charlie Hall. ${ }^{1}$ Marie Curie Hospice, Edinburgh, UK: ${ }^{2}$ Marie Curie Hospice, Glasgow, Scotland, UK; ${ }^{3}$ NHS Fife, UK

10.1136/bmjspcare-2017-00133.97

Background Following recent changes in UK case law, DNACPR decisions must be discussed with patients unless that conversation will cause harm. CPR should not be given if it is an inappropriate treatment. DNACPR conversations are therefore happening more frequently; yet evidence to support staff in knowing how to undertake these conversations is scarce from the patient/caregiver perspective. This integrative literature review aims to identify patient and family experiences of DNACPR conversations.

Methods A search of multiple databases was performed for adult patients and caregivers, from all settings worldwide (2004-2014). Abstracts were reviewed for relevance and quality. First person, retrospective accounts of patient/carer experience of DNACPR conversations were gold standard. Relevant full texts were appraised and recurring themes analysed and tabulated.

Results The initial search identified 559 abstracts. Of these, 46 full texts were deemed relevant including 9 UK and 37 international papers. Only 22 papers were original research with patients or carers who had experienced these conversations. Themes revealed the importance of DNACPR discussions being conducted by someone trusted and the importance of family/carer involvement, as well as perceived concerns about burdening family members. Timing preferences for discussions was variable revealing difficulties in finding the 'right time' to discuss. Discussions held at home or the GP surgery are preferable to discussions during acute admissions to hospital.

Conclusions This review highlights potential discrepancies between patient and carer preferences for DNACPR conversations, and the reality of how these conversations are currently taking place. Public health approaches to tackle the societal taboo around death and dying may encourage people to think about and expect such conversations during their illness trajectories. Discussion of DNACPR decisions with the patient is now a legal requirement, but patient centred care remains paramount. More evidence is essential to bridge these expectations.

\section{P-99 THE USE OF AN ONCOLOGICAL PALLIATIVE DEPRESCRIBING GUIDELINE TO AID RATIONALISING MEDICATIONS IN PATIENTS IN THE LAST SIX MONTHS OF LIFE}

Anil Prabhu, Anna Sutherland, Victoria Bradley, Helen Pegrum. Florence Nightingale Hospice, Aylesbury, UK

\subsection{6/bmjspcare-2017-00133.98}

Background It is widely recognised that large numbers of patients remain on unnecessary medications after transition from a curative to palliative pathway. This is often the result of a lack of vigilance and confidence amongst healthcare professionals when rationalising medications ${ }^{[1]}$. This can negatively impact on quality of life, through 'pill burdens' and 